\title{
Existence of Tripled Fixed Points for a Class of Condensing Operators in Banach Spaces
}

\author{
Vatan Karakaya, ${ }^{1}$ Nour El Houda Bouzara, ${ }^{2}$ Kadri Doğan, ${ }^{1}$ and Yunus Atalan ${ }^{2}$ \\ ${ }^{1}$ Department of Mathematical Engineering, Faculty of Chemistry-Metallurgical, Yildiz Technical University, 34210 Istanbul, Turkey \\ ${ }^{2}$ Department of Mathematics, Faculty of Science and Letters, Yildiz Technical University, 34210 Istanbul, Turkey
}

Correspondence should be addressed to Vatan Karakaya; vkkaya@yildiz.edu.tr

Received 28 May 2014; Accepted 23 June 2014; Published 14 September 2014

Academic Editor: S. A. Mohiuddine

Copyright (c) 2014 Vatan Karakaya et al. This is an open access article distributed under the Creative Commons Attribution License, which permits unrestricted use, distribution, and reproduction in any medium, provided the original work is properly cited.

We give some results concerning the existence of tripled fixed points for a class of condensing operators in Banach spaces. Further, as an application, we study the existence of solutions for a general system of nonlinear integral equations.

\section{Introduction and Preliminaries}

Measures of noncompactness are very useful tools in functional analysis, for instance, in metric fixed point theory and in the theory of operator equations in Banach spaces. The first measure of noncompactness, denoted by $\mu$, was defined and studied by Kuratowski [1] in 1930. In 1955, Banaś and Goebel [2] used the function $\mu$ to prove his fixed point theorem. Darbo's fixed point theorem [2] is a very important generalization of Schauder's fixed point theorem [3] and several authors had used this concept for the resolution of nonlinear equations, some of whom are Aghajani et al. $[4,5]$, Banaś [6], Banaś and Rzepka [7], Mursaleen and Mohiuddine [8], and many others. Recently in [9], Aghajani et al. give a generalization of Darbo's fixed point theorem. Moreover, they present some results on the existence of coupled fixed points for class of condensing operators. In this paper, we generalize these results to obtain the existence of tripled fixed points for the same class of operators.

Throughout this paper, $X$ is assumed to be a Banach space and $\mathrm{BC}\left(\mathbb{R}^{+}\right)$is the space of all real functions defined, bounded and continuous on $\mathbb{R}^{+}$. The family of bounded subset, closure, and closed convex hull of $X$ are denoted by $\mathscr{B}_{X}, \bar{X}$, and $\operatorname{Conv} X$, respectively.

Definition 1 (see [10]). Let $X$ be a Banach space and $\mathscr{B}_{X}$ the family of bounded subset of $X$. A map

$$
\mu: \mathscr{B}_{X} \longrightarrow[0, \infty)
$$

is called measure of noncompactness defined on $X$ if it satisfies the following.

(1) $\mu(A)=0 \Leftrightarrow A$ is a precompact set.

(2) $A \subset B \Rightarrow \mu(A) \leqslant \mu(B)$.

(3) $\mu(A)=\mu(\bar{A}), \forall A \in \mathscr{B}_{X}$.

(4) $\mu(\operatorname{Conv} A)=\mu(A)$.

(5) $\mu(\lambda A+(1-\lambda) B) \leqslant \lambda \mu(A)+(1-\lambda) \mu(B)$, for $\lambda \epsilon$ $[0,1]$.

(6) Let $\left(A_{n}\right)$ be a sequence of closed sets from $\mathscr{B}_{X}$ such that $A_{n+1} \subseteq A_{n},(n \geqslant 1)$, and $\lim _{n \rightarrow \infty} \mu\left(A_{n}\right)=0$. Then, the intersection set $A_{\infty}=\bigcap_{n=1}^{\infty} A_{n}$ is nonempty and $A_{\infty}$ is precompact.

Theorem 2 (see [2]). Let $C$ be a nonempty closed, bounded, and convex subset of $X$. If $T: C \rightarrow C$ is a continuous mapping

$$
\mu(T A) \leqslant k \mu(A), \quad k \in[0,1),
$$

then $T$ has a fixed point.

Theorem 3 (see [9]). Let $C$ be a nonempty closed, bounded, and convex subset of $X$ and $T: C \rightarrow C$ a continuous mapping such that for any subset $A$ of $C$

$$
\mu(T A) \leqslant \beta(\mu(A)) \mu(A),
$$


where $\beta: \mathbb{R}_{+} \rightarrow[0,1)$; that is, $\beta\left(t_{n}\right) \rightarrow 1$ implies $t_{n} \rightarrow 0$. Then, $T$ has at least one fixed point.

The following result is a corollary of the previous theorem.

Corollary 4 (see [9]). Let $C$ be a nonempty closed, bounded, and convex subset of $X$ and $T: C \rightarrow C$ a continuous mapping such that for any subset $A$ of $C$

$$
\mu(T A) \leqslant \varphi(\mu(A)),
$$

where $\varphi: \mathbb{R}_{+} \rightarrow \mathbb{R}_{+}$is a nondecreasing and upper semicontinuous functions; that is, for every $t>0, \varphi(t)<t$. Then, $T$ has at least one fixed point.

Definition 5 (see [11]). A coupled fixed point of a mapping $G: X \times X \rightarrow X$ is an element $(x, y) \in X \times X$ such that $G(x, y)=x$ and $G(y, x)=y$.

Theorem 6 (see [12]). Let $\mu_{1}, \mu_{2}, \ldots, \mu_{n}$ be measures of noncompactness in Banach spaces $E_{1}, E_{2}, \ldots, E_{n}$, (respectively).

Then, the function

$$
\widetilde{\mu}(X)=F\left(\mu_{1}\left(X_{1}\right), \mu_{2}\left(X_{2}\right), \ldots, \mu_{n}\left(X_{n}\right)\right),
$$

defines a measure of noncompactness in $E_{1} \times E_{2} \times \cdots \times E_{n}$, where $X_{i}$ is the natural projection of $X$ on $E_{i}$, for $i=1,2, \ldots, n$, and $F$ is a convex function defined by

$$
F:[0, \infty)^{n} \longrightarrow[0, \infty),
$$

such that

$$
\begin{array}{r}
F\left(x_{1}, x_{2}, \ldots, x_{n}\right)=0 \Longleftrightarrow x_{i}=0, \\
\text { for } i=1,2, \ldots, n .
\end{array}
$$

Remark 7. Aghajani and Sabzali [13] illustrated the previous theorem by the following example. Let the mapping $F$ be as follows:

$$
\begin{array}{r}
F(x, y)=\max \{x, y\}, \quad \text { or } \quad F(x, y)=x+y, \\
\text { for any }(x, y) \in[0, \infty)^{2} .
\end{array}
$$

They showed that

$$
\widetilde{\mu}(X)=\max \left(\mu_{1}\left(X_{1}\right), \mu_{2}\left(X_{2}\right)\right),
$$

or

$$
\widetilde{\mu}(X)=\mu_{1}\left(X_{1}\right)+\mu_{2}\left(X_{2}\right)
$$

defines a measure of noncompactness in the space $E_{1} \times E_{2}$, where, for $i=1,2, \mu_{i}$ are measure of noncompactness in $E_{i}$ and $X_{i}, i=1,2$ are the natural projections of $X$ on $E_{i}$.

Theorem 8 (see [9]). Let $\Omega$ be a nonempty, bounded, closed, and convex subset of a Banach space $E$ and let $\varphi: \mathbb{R}^{+} \rightarrow \mathbb{R}^{+}$. Assume that $\varphi$ is a nondecreasing and upper semicontinuous function. Let $G: \Omega \times \Omega \rightarrow \Omega$ be a continuous operator satisfying

$$
\begin{array}{r}
\mu\left(G\left(X_{1} \times X_{2}\right)\right) \leqslant \varphi\left(\frac{\mu\left(X_{1}\right)+\mu\left(X_{2}\right)}{2}\right), \\
X_{1}, X_{2} \in \Omega,
\end{array}
$$

for any measure of noncompactness $\mu$. Then, $G$ has at least a coupled fixed point.

\section{Main Results}

Definition 9 (see [14]). A tripled $(x, y, z)$ of a mapping $G$ : $X \times X \times X \rightarrow X$ is called a tripled fixed point if

$$
\begin{aligned}
& G(x, y, z)=x, \\
& G(y, x, z)=y, \\
& G(z, y, x)=z .
\end{aligned}
$$

Remark 10. We can notice that by taking

$$
\begin{aligned}
& F(x, y, z)=\max \{x, y, z\}, \\
& \text { for any }(x, y, z) \in[0, \infty)^{3},
\end{aligned}
$$

or

$$
F(x, y, z)=x+y+z, \quad \text { for any }(x, y, z) \in[0, \infty)^{3}
$$

$F$ satisfies the conditions of Theorem 6 . Thus, for a measure of noncompactness $\mu_{i}(i=1,2,3)$, we have that

$$
\tilde{\mu}(X)=\max \left(\mu_{1}\left(X_{1}\right), \mu_{2}\left(X_{2}\right), \mu_{3}\left(X_{3}\right)\right),
$$

or

$$
\widetilde{\mu}(X)=\mu_{1}\left(X_{1}\right)+\mu_{2}\left(X_{2}\right)+\mu_{3}\left(X_{3}\right)
$$

defines a measure of noncompactness in the space $E \times E \times E$ where $X_{i}, i=1,2,3$ are the natural projections of $X$ on $E_{i}$.

So, we obtain the following theorem.

Theorem 11. Let $\Omega$ be a nonempty, bounded, closed, and convex subset of a Banach space $E$ and let $\varphi: \mathbb{R}^{+} \rightarrow \mathbb{R}^{+}$be a nondecreasing and upper semicontinuous function such that $\varphi(t)<t$ for all $t>0$. Then, for any measure of noncompactness $\mu$, the continuous operator $G: \Omega \times \Omega \times \Omega \rightarrow \Omega$ satifying

$$
\begin{array}{r}
\mu\left(G\left(X_{1} \times X_{2} \times X_{3}\right)\right) \leqslant \varphi\left(\frac{\mu\left(X_{1}\right)+\mu\left(X_{2}\right)+\mu\left(X_{3}\right)}{3}\right), \\
X_{1}, X_{2}, X_{3} \in \Omega
\end{array}
$$

has at least a tripled fixed point.

Proof. To prove this theorem, let us define the measure of noncompactness $\tilde{\mu}$ by

$$
\tilde{\mu}(X)=\mu_{1}\left(X_{1}\right)+\mu_{2}\left(X_{2}\right)+\mu_{3}\left(X_{3}\right)
$$

and the mapping $\widetilde{G}: \Omega \times \Omega \times \Omega \rightarrow \Omega$

$$
\widetilde{G}(x, y, z)=(G(x, y, z), G(y, x, z), G(z, y, x)) .
$$


Since

$$
\begin{aligned}
\tilde{\mu}(\widetilde{G}(X)) \leqslant & \tilde{\mu}\left(G\left(X_{1} \times X_{2} \times X_{3}\right) \times G\left(X_{2} \times X_{1} \times X_{3}\right)\right. \\
& \left.\times G\left(X_{3} \times X_{2} \times X_{1}\right)\right) \\
= & \mu\left(G\left(X_{1} \times X_{2} \times X_{3}\right)\right)+\mu\left(G\left(X_{2} \times X_{1} \times X_{3}\right)\right) \\
& +\mu\left(G\left(X_{3} \times X_{2} \times X_{1}\right)\right) \\
\leqslant & \varphi\left(\frac{\mu\left(X_{1}\right)+\mu\left(X_{2}\right)+\mu\left(X_{3}\right)}{3}\right) \\
& +\varphi\left(\frac{\mu\left(X_{1}\right)+\mu\left(X_{2}\right)+\mu\left(X_{3}\right)}{3}\right) \\
& +\varphi\left(\frac{\mu\left(X_{1}\right)+\mu\left(X_{2}\right)+\mu\left(X_{3}\right)}{3}\right) \\
= & 3 \varphi\left(\frac{\mu\left(X_{1}\right)+\mu\left(X_{2}\right)+\mu\left(X_{3}\right)}{3}\right)
\end{aligned}
$$

and $\tilde{\mu}^{\prime}=(1 / 3) \tilde{\mu}$ is a measure of noncompactness, we get

$$
\tilde{\mu}^{\prime}(\widetilde{G}(X)) \leqslant \varphi\left(\widetilde{\mu}^{\prime}(X)\right) .
$$

By Corollary 4, we obtain that $G$ has at least a tripled fixed point.

\section{Applications}

We can see an application of Theorem 11 in the study of existence of solutions for systems of integral equations defined on the Banach space BC $\left(\mathbb{R}^{+}\right)$endowed with the norm

$$
\|x\|=\sup \{|x(t)|: t>0\} .
$$

The measure of noncompactness on $\mathrm{BC}\left(\mathbb{R}^{+}\right)$for a positive fixed $t$ on $\mathscr{B}_{\mathrm{BC}\left(\mathbb{R}^{+}\right)}$is defined as follows:

$$
\mu(X)=\omega_{0}(X)+\lim \sup _{t \rightarrow \infty} \operatorname{diam} X(t)
$$

such that

$$
\begin{array}{r}
\operatorname{diam} X(t)=\sup \{|x(t)-y(t)|: x, y \in X\}, \\
\text { where } X(t)=\{x(t): x \in X\} .
\end{array}
$$

Before defining $\omega_{0}(X)$, we need first to introduce the modulus of continuity.

Let $x \in X$ and $\epsilon>0$;

$$
\omega^{T}(x, \epsilon)=\sup \{|x(t)-x(s)|: t, s \in[0, T],|t-s| \leqslant \epsilon\},
$$$$
\text { for } T>0 \text {, }
$$

is the modulus of continuity of $x$ on $[0, T]$ and let

$$
\begin{aligned}
\omega^{T}(X, \epsilon) & =\sup \left\{\omega^{T}(x, \epsilon): x \in X\right\}, \\
\omega_{0}^{T}(X) & =\lim _{\epsilon \rightarrow 0} \omega^{T}(X, \epsilon), \\
\omega_{0}(X) & =\lim _{T \rightarrow \infty} \omega_{0}^{T}(X) .
\end{aligned}
$$

Assume that

(i) $\xi, \eta, q: \mathbb{R}_{+} \rightarrow \mathbb{R}_{+}$are continuous and $\xi(t) \rightarrow \infty$ as $t \rightarrow \infty$

(ii) the function $\psi: \mathbb{R} \rightarrow \mathbb{R}$ is continuous and there exist positive $\delta, \alpha$ such that

$$
\left|\psi\left(t_{1}\right)-\psi\left(t_{2}\right)\right| \leqslant \delta\left|t_{1}-t_{2}\right|^{\alpha}
$$

for any $t_{1}, t_{2} \in \mathbb{R}_{+}$;

(iii) $f: \mathbb{R}_{+} \times \mathbb{R} \times \mathbb{R} \times \mathbb{R} \times \mathbb{R} \times \mathbb{R} \rightarrow \mathbb{R}$ is continuous and there exists a nondecareasing continuous function $\Phi$ : $\mathbb{R} \rightarrow \mathbb{R}$ with $\Phi(0)=0$, so that

$$
\begin{aligned}
& \left|f\left(t, x_{1}, x_{2}, x_{3}, x_{4}\right)-f\left(t, y_{1}, y_{2}, y_{3}, y_{4}\right)\right| \\
& \leqslant \frac{1}{2}\left(\varphi\left(\left|x_{1}-y_{1}\right|+\left|x_{2}-y_{2}\right|+\left|x_{3}-y_{3}\right|\right)\right) \\
& \quad+\Phi\left(\left|x_{4}-y_{4}\right|\right) ;
\end{aligned}
$$

(iv) the function defined by $|f(t, 0,0,0,0)|$ is bounded on $\mathbb{R}_{+}$; that is,

$$
M_{1}=\sup \left\{f(t, 0,0,0,0): t \in \mathbb{R}_{+}\right\}<\infty
$$

(v) $h: \mathbb{R}_{+} \times \mathbb{R}_{+} \times \mathbb{R} \times \mathbb{R} \rightarrow \mathbb{R}$ is a continuous function and there exists a positive solution $r_{0}$ of the inequality

$$
\frac{1}{3} \varphi(3 r)+M_{1}+\Phi(\delta D) \leqslant r
$$

where $D$ is positive constant defined by the equality

$$
\begin{gathered}
D=\sup \left\{\left|\int_{0}^{q(t)}(t, s, x(\eta(s)), y(\eta(s)), z(\eta(s))) d s\right|:\right. \\
\left.t, s \in \mathbb{R}_{+}, x, y, z \in \mathrm{BC}\left(\mathbb{R}_{+}\right)\right\},
\end{gathered}
$$

$$
\begin{aligned}
\lim _{\epsilon \rightarrow \infty} \int_{0}^{q(t)}[ & h(t, s, x(\eta(s)), y(\eta(s)), z(\eta(s))) \\
& -h(t, s, u(\eta(s)), v(\eta(s)), w(\eta(s)))] d s=0,
\end{aligned}
$$

uniformly with respect to $x, y, z, u, v, w \in \mathrm{BC}\left(\mathbb{R}_{+}\right)$. 
Theorem 12. Suppose that (i)-(v) hold; then the system of integral equations

$$
\begin{aligned}
& x(t) \\
& =f(t, x(\xi(t)), y(\xi(t)), z(\xi(t)), \\
& \left.\psi\left(\int_{0}^{q(t)} h(t, s, x(\eta(s)), y(\eta(s)), z(\eta(s))) d s\right)\right), \\
& y(t) \\
& =f(t, y(\xi(t)), x(\xi(t)), z(\xi(t)), \\
& \left.\psi\left(\int_{0}^{q(t)} h(t, s, y(\eta(s)), x(\eta(s)), z(\eta(s))) d s\right)\right), \\
& z(t) \\
& =f(t, z(\xi(t)), y(\xi(t)), x(\xi(t)), \\
& \left.\psi\left(\int_{0}^{q(t)} h(t, s, z(\eta(s)), y(\eta(s)), x(\eta(s))) d s\right)\right)
\end{aligned}
$$

has at least one solution in the space $B C\left(\mathbb{R}_{+}\right) \times B C\left(\mathbb{R}_{+}\right) \times$ $B C\left(\mathbb{R}_{+}\right)$.

Proof. Let $G: \mathrm{BC}\left(\mathbb{R}_{+}\right) \times \mathrm{BC}\left(\mathbb{R}_{+}\right) \times \mathrm{BC}\left(\mathbb{R}_{+}\right) \rightarrow \mathrm{BC}\left(\mathbb{R}_{+}\right)$be an operator defined by

$$
\begin{aligned}
& G(x, y, z)(t) \\
& =f(t, x(\xi(t)), y(\xi(t)), z(\xi(t)), \\
& \left.\quad \psi\left(\int_{0}^{q(t)} h(t, s, x(\eta(s)), y(\eta(s)), z(\eta(s))) d s\right)\right) .
\end{aligned}
$$

For $(x, y, z) \in \mathrm{BC}\left(\mathbb{R}_{+}\right) \times \mathrm{BC}\left(\mathbb{R}_{+}\right) \times \mathrm{BC}\left(\mathbb{R}_{+}\right)$, let

$$
\begin{gathered}
\|(x, y, z)\|_{\mathrm{BC}\left(\mathbb{R}_{+}\right) \times \mathrm{BC}\left(\mathbb{R}_{+}\right) \times \mathrm{BC}\left(\mathbb{R}_{+}\right)} \\
=\|x\|_{\infty}+\|y\|_{\infty}+\|z\|_{\infty} .
\end{gathered}
$$

We can easily prove that the solution of (32) in $\mathrm{BC}\left(\mathbb{R}_{+}\right) \times$ $\mathrm{BC}\left(\mathbb{R}_{+}\right) \times \mathrm{BC}\left(\mathbb{R}_{+}\right)$is equivalent to the tripled fixed point of $G$.
Obviously, $G(x, y, z)(t)$ is continuous for any $(x, y, z) \epsilon$ $\mathrm{BC}\left(\mathbb{R}_{+}\right) \times \mathrm{BC}\left(\mathbb{R}_{+}\right) \times \mathrm{BC}\left(\mathbb{R}_{+}\right)$. Hence, we have

$$
\begin{aligned}
& |G(x, y, z)(t)| \\
& \leqslant \mid f(t, x(\xi(t)), y(\xi(t)), z(\xi(t)) \\
& \left.\quad \psi\left(\int_{0}^{q(t)} h(t, s, x(\eta(s)), y(\eta(s)), z(\eta(s))) d s\right)\right) \\
& \quad+|f(t, 0,0,0,0)| \\
& \leqslant \frac{1}{2} \varphi(|x(\xi(t))|+|y(\xi(t))|+|y(\xi(t))|) \\
& \quad+\Phi\left(\left|\psi\left(\int_{0}^{q(t)} h(t, s, x(\eta(s)), y(\eta(s)), z(\eta(s))) d s\right)\right|\right) \\
& +|f(t, 0,0,0,0)| \\
& \quad+|f(t, 0,0,0,0)| \cdot \\
& \leqslant
\end{aligned}
$$

Then, by (29) and (30), we get

$$
\begin{aligned}
& \|G(x, y, z)\|_{\infty} \\
& \quad \leqslant \frac{1}{3} \varphi\left(\|x\|_{\infty}+\|y\|_{\infty}+\|z\|_{\infty}\right)+M_{1}+\Phi(\delta D) \leqslant r_{0} .
\end{aligned}
$$

So, we obtain

$$
G\left(\bar{B}_{r_{0}} \times \bar{B}_{r_{0}} \times \bar{B}_{r_{0}}\right) \subset \bar{B}_{r_{0}}
$$

Now, we prove that $G: \bar{B}_{r_{0}} \times \bar{B}_{r_{0}} \times \bar{B}_{r_{0}} \rightarrow \bar{B}_{r_{0}}$ is continuous. Let $(x, y, z),(u, v, w) \in \bar{B}_{r_{0}} \times \bar{B}_{r_{0}} \times \bar{B}_{r_{0}}$ such that, for $\varepsilon>0$,

$$
\|(x, y, z)-(u, v, w)\|_{\bar{B}_{r_{0}} \times \bar{B}_{r_{0}} \times \bar{B}_{r_{0}}}<\epsilon .
$$


Then,

$$
\begin{aligned}
& |G(x, y, z)(t)-G(u, v, w)(t)| \\
& =\mid f(t, x(\xi(t)), y(\xi(t)), z(\xi(t)), \\
& \left.\psi\left(\int_{0}^{q(t)} h(t, s, x(\eta(s)), y(\eta(s)), z(\eta(s))) d s\right)\right) \\
& -f(t, x(\xi(t)), y(\xi(t)), z(\xi(t)), \\
& \quad \psi\left(\int_{0}^{q(t)} h(t, s, u(\eta(s)), v(\eta(s)),\right. \\
& \leqslant \frac{1}{2} \varphi(|x(\xi(t)-u(\xi(t)))|+|y(\xi(t))-v(\xi(t))| \\
& +|z(\xi(t))-w(\xi(t))|) \\
& +\Phi\left(\psi\left(\int_{0}^{q(t)} h(t, s, x(\eta(s)), y(\eta(s)), z(\eta(s))) d s\right)\right. \\
& \left.\quad-\psi\left(\int_{0}^{q(t)} h(t, s, u(\eta(s)), v(\eta(s)), w(\eta(s))) d s\right)\right) \\
& \left.+\infty \int_{0}^{q(t)} h(t, s, u(\eta(s)), v(\eta(s)), w(\eta(s))) d s \mid\right) . \\
& \leqslant \frac{1}{2} \varphi(|x(\xi(t)-u(\xi(t)))|+|y(\xi(t))-v(\xi(t))|) \\
& +|z(\xi(t))-w(\xi(t))|) \\
& +\delta\left(\int_{0}^{q(t)} h(t, s, x(\eta(s)), y(\eta(s)), z(\eta(s))) d s\right.
\end{aligned}
$$

Using condition (iii) and (29), there exists $T>0$ such that if $t>T$, then

$$
\begin{aligned}
& \Phi\left(\delta\left|\int_{0}^{q(t)} h(t, s, x(\eta(s)), y(\eta(s), z(\eta(s))) d s)\right|^{\alpha}\right) \\
& \quad \leqslant \frac{1}{3} \epsilon,
\end{aligned}
$$

for any $x, y, z \in \mathrm{BC}\left(\mathbb{R}_{+}\right)$. We notice two cases.

Case 1. If $t>T$, then from (39) and (40)

$$
|G(x, y, z)(t)-G(u, v, w)(t)| \leqslant \frac{1}{3} \varphi(\epsilon)+\frac{1}{3} \epsilon .
$$

Case 2. Similarly, for $t \in[0, T]$, we have

$$
\begin{aligned}
&|G(x, y, z)(t)-G(u, v, w)(t)| \\
& \leqslant \frac{1}{2} \varphi(|x(\xi(t)-u(\xi(t)))|+|y(\xi(t))-v(\xi(t))|) \\
&+|z(\xi(t))-w(\xi(t))|)
\end{aligned}
$$

$$
\begin{aligned}
& +\Phi\left(\delta \mid \int_{0}^{q(t)} h(t, s, x(\eta(s)), y(\eta(s)), z(\eta(s))) d s\right. \\
& \left.\quad-\left.\quad \int_{0}^{q(t)} h(t, s, u(\eta(s)), v(\eta(s)), w(\eta(s))) d s\right|^{\alpha}\right) \\
& \leqslant \frac{1}{2} \varphi(\epsilon)+\Phi\left(\delta\left(q_{T} \beta(\epsilon)\right)^{\alpha}\right) \\
& <\frac{1}{2} \epsilon+\Phi\left(\delta\left(q_{T} \beta(\epsilon)\right)^{\alpha}\right),
\end{aligned}
$$

where $q_{T}=\sup \{q(t): t \in[0, T]\}$, and

$$
\begin{gathered}
\beta(\epsilon)=\sup \{|h(t, s, x, y, z)-h(t, s, u, v, w)|: \\
t \in[0, \mathrm{~T}], s \in\left[0, q_{T}\right], \\
x, y, z, u, v, w \in\left[-r_{0}, r_{0}\right] \\
\|(x, y, z)-(u, v, w)\|<\epsilon\} .
\end{gathered}
$$

Since $\beta$ is continuous on $[0, T] \times\left[0, q_{T}\right] \times\left[-r_{0}, r_{0}\right] \times\left[-r_{0}, r_{0}\right]$, we have $\beta(\epsilon) \rightarrow 0$ and $\epsilon \rightarrow 0$. Thus, using (iii), we get

$$
\Phi\left(\delta\left(q_{T} \beta(\epsilon)\right)^{\alpha}\right) \longrightarrow 0, \quad \text { as } \epsilon \longrightarrow 0 .
$$

Finally, from (42) and (41), we conclude that $G$ is a continuous function from $\bar{B}_{r_{0}} \times \bar{B}_{r_{0}} \times \bar{B}_{r_{0}}$ into $\bar{B}_{r_{0}}$.

Now, we show that the map $G$ satisfies all the conditions of Theorem 11. To do this, for an arbitrary fixed $T>0$ and $\epsilon>$ 0 , assume that $X_{1}, X_{2}$, and $X_{3}$ are nonempty chosen subsets of $\bar{B}_{r_{0}}$ and $t_{1}, t_{2} \in[0, T]$, with $\left|t_{2}-t_{1}\right| \leqslant \epsilon$. Without loss of generality, let

$$
q\left(t_{1}\right)<q\left(t_{2}\right) .
$$

For an arbitrary $(x, y, z) \in X_{1} \times X_{2} \times X_{3}$,

$$
\begin{aligned}
& \left|G(x, y, z)\left(t_{1}\right)-G(x, y, z)\left(t_{2}\right)\right| \\
& \leqslant \mid f\left(t_{1}, x\left(\xi\left(t_{1}\right)\right), y\left(\xi\left(t_{1}\right)\right), z\left(\xi\left(t_{1}\right)\right),\right. \\
& \left.\psi\left(\int_{0}^{q\left(t_{1}\right)} h\left(t_{1}, s, x(\eta(s)), y(\eta(s)), z(\eta(s))\right) d s\right)\right) \\
& -f\left(t_{1}, x\left(\xi\left(t_{2}\right)\right), y\left(\xi\left(t_{2}\right)\right), z\left(\xi\left(t_{2}\right)\right),\right. \\
& \psi\left(\int _ { 0 } ^ { q ( t _ { 1 } ) } h \left(t_{1}, s, x(\eta(s)),\right.\right. \\
& y(\eta(s)), z(\eta(s))) d s)) \mid
\end{aligned}
$$




$$
\begin{aligned}
& +\mid f\left(t_{1}, x\left(\xi\left(t_{2}\right)\right), y\left(\xi\left(t_{2}\right)\right), z\left(\xi\left(t_{2}\right)\right),\right. \\
& \left.\psi\left(\int_{0}^{q\left(t_{1}\right)} h\left(t_{1}, s, x(\eta(s)), y(\eta(s)), z(\eta(s))\right) d s\right)\right) \\
& -f\left(t_{2}, x\left(\xi\left(t_{2}\right)\right), y\left(\xi\left(t_{2}\right)\right), z\left(\xi\left(t_{2}\right)\right),\right. \\
& \psi\left(\int _ { 0 } ^ { q ( t _ { 1 } ) } h \left(t_{1}, s, x(\eta(s)),\right.\right. \\
& y(\eta(s)), z(\eta(s))) d s)) \\
& +\mid f\left(t_{2}, x\left(\xi\left(t_{2}\right)\right), y\left(\xi\left(t_{2}\right)\right), z\left(\xi\left(t_{2}\right)\right),\right. \\
& \left.\psi\left(\int_{0}^{q\left(t_{1}\right)} h\left(t_{1}, s, x(\eta(s)), y(\eta(s)), z(\eta(s))\right) d s\right)\right) \\
& -f\left(t_{2}, x\left(\xi\left(t_{2}\right)\right), y\left(\xi\left(t_{2}\right)\right), z\left(\xi\left(t_{2}\right)\right),\right. \\
& \psi\left(\int _ { 0 } ^ { q ( t _ { 1 } ) } h \left(t_{1}, s, x(\eta(s))\right.\right. \\
& y(\eta(s)), z(\eta(s))) d s)) \\
& +\mid f\left(t_{2}, x\left(\xi\left(t_{2}\right)\right), y\left(\xi\left(t_{2}\right)\right), z\left(\xi\left(t_{2}\right)\right),\right. \\
& \left.\psi\left(\int_{0}^{q\left(t_{1}\right)} h\left(t_{1}, s, x(\eta(s)), y(\eta(s)), z(\eta(s))\right) d s\right)\right) \\
& -f\left(t_{2}, x\left(\xi\left(t_{2}\right)\right), y\left(\xi\left(t_{2}\right)\right), z\left(\xi\left(t_{2}\right)\right),\right. \\
& \psi\left(\int _ { 0 } ^ { q ( t _ { 2 } ) } h \left(t_{2}, s, x(\eta(s))\right.\right. \\
& y(\eta(s)), z(\eta(s))) d s)) \\
& \leqslant \frac{1}{3} \varphi\left(\left|x\left(\xi\left(t_{1}\right)-x\left(\xi\left(t_{2}\right)\right)\right)\right|+\left|y\left(\xi\left(t_{1}\right)-y\left(\xi\left(t_{2}\right)\right)\right)\right|\right. \\
& \left.+\left|z\left(\xi\left(t_{1}\right)-z\left(\xi\left(t_{2}\right)\right)\right)\right|+\omega_{r_{0}, D_{1}}^{T}(f, \epsilon)\right) \\
& +\Phi\left(\delta \mid \int_{0}^{q\left(t_{2}\right)}\left[h\left(t_{2}, s, x(\eta(s)), y(\eta(s)), z(\eta(s))\right)\right.\right. \\
& -h\left(t_{1}, s, x(\eta(s)), y(\eta(s))\right. \\
& \left.z(\eta(s)))]\left.d s\right|^{\alpha}\right)
\end{aligned}
$$$$
+\Phi\left(\delta\left|\int_{q\left(t_{1}\right)}^{q\left(t_{2}\right)} h\left(t_{1}, s, x(\eta(s)), y(\eta(s)), z(\eta(s))\right) d s\right|^{\alpha}\right)
$$$$
\begin{aligned}
\leqslant & \frac{1}{3} \varphi\left(\omega^{T}\left(x, \omega^{T}(\xi, \epsilon)\right)+\omega^{T}\left(y, \omega^{T}(\xi, \epsilon)\right)\right. \\
& \left.+\omega^{T}\left(z, \omega^{T}(\xi, \epsilon)\right)+\omega_{r_{0}, D_{1}}^{T}(f, \epsilon)\right) \\
& +\Phi\left(\delta\left(q_{T} \omega_{r_{0}}^{T}(h, \epsilon)\right)^{\alpha}\right)+\Phi\left(\delta\left(U_{r_{0}}^{T} \omega^{T}(q, \epsilon)\right)^{\alpha}\right)
\end{aligned}
$$

where

$$
\begin{aligned}
& \omega^{T}(\xi, \epsilon)=\sup \left\{\left|\left(\xi\left(t_{2}\right)-\xi\left(t_{1}\right)\right)\right|: t_{1}, t_{2} \leqslant \epsilon,\left|t_{2}-t_{1}\right| \leqslant \epsilon\right\}, \\
& \omega^{T}\left(x, \omega^{T}(\xi, \epsilon)\right)=\sup \left\{\left|\left(x\left(t_{2}\right)-x\left(t_{1}\right)\right)\right|: t_{1}, t_{2} \in[0, T],\right. \\
& \left.\left|t_{2}-t_{1}\right| \leqslant \omega^{T}(\xi, \epsilon)\right\} \\
& D_{1}=q_{T} \sup \left\{|h(t, s, x, y, z)|, t \in[0, T], s \in\left[0, q_{T}\right]\right. \text {, } \\
& \left.x, y, z \in\left[-r_{0}, r_{0}\right]\right\} \\
& \omega_{r_{0}, D_{1}}^{T}(f, \epsilon)=\sup \left\{\left|f\left(t_{2}, x, y, z, d\right)-f\left(t_{1}, x, y, z, d\right)\right|,\right. \\
& t_{1}, t_{2} \in[0, T] \\
& \left|t_{2}-t_{1}\right| \leqslant \epsilon, x, y, z \in\left[-r_{0}, r_{0}\right] \text {, } \\
& \left.d \in\left[-D_{1}, D_{1}\right]\right\} \text {, } \\
& \omega_{r_{0}}^{T}(f, \epsilon)=\sup \left\{\left|h\left(t_{2}, s, x, y, z\right)-f\left(t_{1}, s, x, y, z\right)\right|\right. \text {, } \\
& t_{1}, t_{2} \in[0, T],\left|t_{2}-t_{1}\right| \leqslant \epsilon, \\
& \left.s \in\left[0, q_{T}\right], x, y, z \in\left[-r_{0}, r_{0}\right]\right\}, \\
& U_{r_{0}}^{T}=\sup \left\{\left|h\left(t_{1}, s, x, y, z\right)\right|: t_{1} \in[0, T]\right. \text {, } \\
& \left.s \in\left[0, q_{T}\right], x, y, z \in\left[-r_{0}, r_{0}\right]\right\},
\end{aligned}
$$

we obtain

$$
\begin{aligned}
\omega^{T}( & \left.G\left(X_{1} \times X_{2} \times X_{3}\right), \epsilon\right) \\
\leqslant & \frac{1}{3} \varphi\left(\omega^{T}\left(X_{1}, \omega^{T}(\xi, \epsilon)\right)+\omega^{T}\left(X_{2}, \omega^{T}(\xi, \epsilon)\right)\right. \\
& \left.+\omega^{T}\left(X_{3}, \omega^{T}(\xi, \epsilon)\right)\right)+\omega_{r_{0}, D_{1}}^{T}(f, \epsilon) \\
& +\Phi\left(\delta\left(q_{T} \omega_{r_{0}}^{T}(h, \epsilon)\right)^{\alpha}\right)+\Phi\left(\delta\left(U_{r_{0}}^{T} \omega^{T}(q, \epsilon)\right)^{\alpha}\right) .
\end{aligned}
$$

Further, by the uniform continuity of $f$ and $h$ on the compact sets $[0, T] \times\left[-r_{0}, r_{0}\right] \times\left[-r_{0}, r_{0}\right] \times\left[-r_{0}, r_{0}\right] \times\left[-D_{1}, D_{1}\right]$ and $[0, T] \times\left[0, q_{T}\right] \times\left[-r_{0}, r_{0}\right] \times\left[-r_{0}, r_{0}\right] \times\left[-r_{0}, r_{0}\right]$, respectively, we get $\omega_{r_{0}, D_{1}}^{T}(f, \epsilon) \rightarrow 0$ and $\omega_{r_{0}}^{T}(h, \epsilon) \rightarrow 0$ as $\epsilon \rightarrow 0$. 
Moreover, $\Phi$ is a nondecreasing continuous function with $\Phi(0)=0$ and (iii), and we obtain

$$
\begin{aligned}
\Phi\left(\delta\left(q_{T} \omega_{r_{0}}^{T}(h, \epsilon)\right)^{\alpha}\right)+\Phi\left(\delta\left(U_{r_{0}}^{T} \omega^{T}(q, \epsilon)\right)^{\alpha}\right) & \longrightarrow 0 \\
\epsilon & \longrightarrow 0
\end{aligned}
$$

By (48), we get

$$
\begin{aligned}
& \omega_{0}^{T}\left(G\left(X_{1} \times X_{2} \times X_{3}\right)\right) \\
& \quad \leqslant \frac{1}{3} \varphi\left(\omega_{0}^{T}\left(X_{1}\right)+\omega_{0}^{T}\left(X_{2}\right)+\omega_{0}^{T}\left(X_{3}\right)\right) .
\end{aligned}
$$

Taking the limit $T \rightarrow \infty$ in (50), we obtain

$$
\begin{aligned}
\omega_{0} & \left(G\left(X_{1} \times X_{2} \times X_{3}\right)\right) \\
& \leqslant \frac{1}{3} \varphi\left(\omega_{0}\left(X_{1}\right)+\omega_{0}\left(X_{2}\right)+\omega_{0}\left(X_{3}\right)\right) .
\end{aligned}
$$

Then, for arbitrary $(x, y, z),(u, v, w) \in X_{1} \times X_{2} \times X_{3}$, and $t \in \mathbb{R}_{+}$, we have

$$
\begin{aligned}
& |G(x, y, z)(t)-G(u, v, w)(t)| \\
& \leqslant \frac{1}{3} \varphi(|x(\xi(t))-u(\xi(t))|+|y(\xi(t))-v(\xi(t))| \\
& +|z(\xi(t))-w(\xi(t))|) \\
& +\Phi\left(\delta \mid \int_{0}^{q(t)}[h(t, s, x(\eta(s)), y(\eta(s)), z(\eta(s)))\right. \\
& -h(t, s, u(\eta(s)), \\
& \left.\quad v(\eta(s)), w(\eta(s)))]\left.d s\right|^{\alpha}\right) \\
& \leqslant \frac{1}{2} \varphi\left(\operatorname{diam} X_{1}(\xi(t))+\operatorname{diam} X_{2}(\xi(t))+\operatorname{diam} X_{3}(\xi(t))\right) \\
& +\Phi\left(\delta \mid \int_{0}^{q(t)}[h(t, s, x(\eta(s)), y(\eta(s)), z(\eta(s)))\right. \\
& -h(t, s, u(\eta(s)), \\
& \left.v(\eta(s)), w(\eta(s)))]\left.d s\right|^{\alpha}\right)
\end{aligned}
$$

Since $(x, y, z),(u, v, w)$, and $t$ are arbitrary in (52),

$$
\begin{aligned}
& \operatorname{diam} G\left(X_{1} \times X_{2} \times X_{3}\right)(t) \\
& \leqslant \frac{1}{3} \varphi\left(\operatorname{diam} X_{1}(\xi(t))+\operatorname{diam} X_{2}(\xi(t))+\operatorname{diam} X_{3}(\xi(t))\right) \\
& +\Phi\left(\delta \mid \int_{0}^{q(t)}[h(t, s, x(\eta(s)), y(\eta(s)), z(\eta(s)))\right. \\
& -h(t, s, u(\eta(s)), v(\eta(s)), \\
& w(\eta(s)))(t, s, x(\eta(s)), \\
& \left.y(\eta(s)), z(\eta(s)))]\left.d s\right|^{\alpha}\right) .
\end{aligned}
$$

Taking again $T \rightarrow \infty$ in (53), we obtain

limsup $\sup _{t \rightarrow \infty} \operatorname{diam} G\left(X_{1} \times X_{2} \times X_{3}\right)(t)+\omega_{0}$

$$
\begin{gathered}
\leqslant \frac{1}{3} \varphi\left(\lim \sup _{t \rightarrow \infty} \operatorname{diam} X_{1}(\xi(t))+\lim \sup _{t \rightarrow \infty} \operatorname{diam} X_{2}(\xi(t))\right. \\
\left.+\lim \sup _{t \rightarrow \infty} \operatorname{diam} X_{3}(\xi(t))\right) .
\end{gathered}
$$

We conclude from (51) and (54) that

$$
\begin{gathered}
\lim _{t \rightarrow \infty} \sup _{t \rightarrow \infty}\left(G\left(X_{1} \times X_{2} \times X_{3}\right)\right)(t)+\omega_{0}\left(G\left(X_{1} \times X_{2} \times X_{3}\right)\right) \\
\leqslant \frac{1}{3} \varphi\left(\lim \sup _{t \rightarrow \infty} \operatorname{diam} X_{1}(\xi(t))+\lim \sup _{t \rightarrow \infty} \operatorname{diam} X_{2}(\xi(t))\right. \\
\left.\quad+\lim \sup _{t \rightarrow \infty} \operatorname{diam} X_{3}(\xi(t))\right) \\
+\frac{1}{3} \varphi\left(\omega_{0}\left(X_{1}\right)+\omega_{0}\left(X_{2}\right)+\omega_{0}\left(X_{3}\right)\right) .
\end{gathered}
$$

Since $\varphi$ is a concave function, (55) implies

$$
\begin{aligned}
\lim \sup _{t \rightarrow \infty} & \operatorname{diam} G\left(X_{1} \times X_{2} \times X_{3}\right)(t) \\
& +\omega_{0}\left(G\left(X_{1} \times X_{2} \times X_{3}\right)\right) \\
\leqslant & \varphi\left(\frac{1}{3}\left[\lim \sup _{t \rightarrow \infty} \operatorname{diam} X_{1}(\xi(t))+\omega_{0}\left(X_{1}\right)\right]\right) \\
& +\varphi\left(\frac{1}{3}\left[\lim \sup _{t \rightarrow \infty} \operatorname{diam} X_{2}(\xi(t))+\omega_{0}\left(X_{2}\right)\right]\right) \\
& +\varphi\left(\frac{1}{3}\left[\lim \sup _{t \rightarrow \infty} \operatorname{diam} X_{3}(\xi(t))+\omega_{0}\left(X_{3}\right)\right]\right) .
\end{aligned}
$$

Finally, since $\mu$ is defined by

$$
\mu(X)=\omega_{0}(X)+\lim \sup _{t \rightarrow \infty} \operatorname{diam} X(t),
$$


we get

$$
\begin{aligned}
& \mu\left(G\left(X_{1} \times X_{2} \times X_{3}\right)\right) \\
& \quad \leqslant \varphi\left(\frac{\mu\left(X_{1}\right)+\mu\left(X_{2}\right)+\mu\left(X_{3}\right)}{3}\right) .
\end{aligned}
$$

Hence, by Theorem 11, $T$ has at least a tripled fixed point in $\mathrm{BC}\left(\mathbb{R}_{+}\right) \times \mathrm{BC}\left(\mathbb{R}_{+}\right) \times \mathrm{BC}\left(\mathbb{R}_{+}\right)$.

Example 1. We consider the following system of integral equations

$$
\begin{aligned}
& x(t)=\frac{1}{3+t^{2}} x(t)+y(t)+z(t) \\
& +\int_{0}^{T}(x(s) s|\sin y(t)||\cos z(t)| \\
& +e^{s}\left(1+x^{2}(s)\right)\left(1+\sin ^{2} y(s)\right) \\
& \left.\cdot\left(1+\cos ^{2} z(s)\right)\right) \\
& \cdot\left(e^{t}\left(1+x^{2}(s)\right)\left(1+\sin ^{2} y(s)\right)\right. \\
& \left.\cdot\left(1+\cos ^{2} z(s)\right)\right)^{-1} d s, \\
& y(t)=\frac{1}{3+t^{2}} y(t)+x(t)+z(t) \\
& +\int_{0}^{T}(y(s) s|\sin x(t)||\cos z(t)| \\
& +e^{s}\left(1+y^{2}(s)\right)\left(1+\sin ^{2} x(s)\right) \\
& \left.\cdot\left(1+\cos ^{2} z(s)\right)\right) \\
& \cdot\left(e^{t}\left(1+y^{2}(s)\right)\left(1+\sin ^{2} x(s)\right)\right. \\
& \left.\cdot\left(1+\cos ^{2} z(s)\right)\right)^{-1} d s, \\
& z(t)=\frac{1}{3+t^{2}} z(t)+y(t)+x(t) \\
& +\int_{0}^{T}(z(s) s|\sin y(t)||\cos x(t)| \\
& +e^{s}\left(1+z^{2}(s)\right)\left(1+\sin ^{2} y(s)\right) \\
& \left.\cdot\left(1+\cos ^{2} x(s)\right)\right) \\
& \cdot\left(e^{t}\left(1+z^{2}(s)\right)\left(1+\sin ^{2} y(s)\right)\right. \\
& \left.\cdot\left(1+\cos ^{2} x(s)\right)\right)^{-1} d s .
\end{aligned}
$$

We notice that by taking

$$
\begin{gathered}
f(t, x, y, z, p)=\frac{1}{3+t^{2}} x+\frac{1}{3} y+\frac{1}{3} z+p, \\
h(t, s, x, y, z) \\
=\frac{x s|\sin y||\cos z|+e^{s}\left(1+x^{2}\right)\left(1+\sin ^{2} y\right)\left(1+\cos ^{2} z\right)}{e^{t}\left(1+x^{2}\right)\left(1+\sin ^{2} y\right)\left(1+\cos ^{2} z\right)} \\
\eta(t)=\xi(t)=q(t)=\Psi(t)=\Phi(t)=t, \\
\varphi(t)=t-3,
\end{gathered}
$$

we get the system integral equations (32).

To solve this system, we need to verify conditions (i) $-(\mathrm{v})$.

Obviously, $\xi, \eta, q: \mathbb{R}_{+} \rightarrow \mathbb{R}_{+}$are continuous and $\xi \rightarrow$ $\infty$ as $t \rightarrow \infty$. Further, the function $\psi: \mathbb{R} \rightarrow \mathbb{R}$ is continuous for $\delta=\alpha=1$, and we have

$$
\left|\psi\left(t_{1}\right)-\psi\left(t_{2}\right)\right| \leqslant \delta\left|t_{1}-t_{2}\right|^{\alpha},
$$

for any $t_{1}, t_{2} \in \mathbb{R}_{+}$. Conditions (i) and (ii) hold.

Now, let

$$
\begin{aligned}
& |f(t, x, y, z, p)-f(t, u, v, w, \rho)| \\
& =\left|\frac{1}{3+t^{2}} x+\frac{1}{3} y+\frac{1}{3} z+p-\left(\frac{1}{3+t^{2}} u+\frac{1}{3} v+\frac{1}{3} w+\rho\right)\right| \\
& \leqslant \frac{1}{3}[|x-u|+|y-v|+|z-w|]+|p-\rho| \\
& =\frac{1}{3} \varphi(|x-u|+|y-v|+|z-w|)+\Phi(|p-\rho|) .
\end{aligned}
$$

Then, (iii) also holds.

Moreover,

$$
M_{1}=\sup \left|\left\{f(t, 0,0,0,0): t \in \mathbb{R}_{+}\right\}\right|=0 ;
$$

then, (iv) is valid.

Let us verify the last condition (v). First,

$$
\begin{aligned}
& |h(t, s, x, y, z)-h(t, s, u, v, w)| \\
& =\mid \frac{x s|\sin y||\cos z|+e^{s}\left(1+x^{2}\right)\left(1+\sin ^{2} y\right)\left(1+\cos ^{2} z\right)}{e^{t}\left(1+x^{2}\right)\left(1+\sin ^{2} y\right)\left(1+\cos ^{2} z\right)} \\
& -\frac{u s|\sin v||\cos w|+e^{s}\left(1+u^{2}\right)\left(1+\sin ^{2} v\right)\left(1+\cos ^{2} w\right)}{e^{t}\left(1+u^{2}\right)\left(1+\sin ^{2} v\right)\left(1+\cos ^{2} w\right)} \mid \\
& =\mid \frac{x s|\sin y||\cos z|}{e^{t}\left(1+x^{2}\right)\left(1+\sin ^{2} y\right)\left(1+\cos ^{2} z\right)} \\
& -\frac{u s|\sin v||\cos w|}{e^{t}\left(1+u^{2}\right)\left(1+\sin ^{2} v\right)\left(1+\cos ^{2} w\right)} \mid \\
& \leqslant\left|\frac{x}{1+x^{2}} \frac{s}{e^{t}}-\frac{u}{1+u^{2}} \frac{s}{e^{t}}\right| \leqslant \frac{1}{2} \frac{s}{e^{t}}+\frac{1}{2} \frac{s}{e^{t}} \leqslant \frac{s}{e^{t}} .
\end{aligned}
$$


Hence,

$$
\begin{aligned}
& \lim _{t \rightarrow \infty} \int_{0}^{t} \mid h(t, s, x(\eta(s)), y(\eta(s)), z(\eta(s))) \\
& \quad-h(t, s, u(\eta(s)), v(\eta(s)), w(\eta(s))) \mid d s \\
& \leqslant \lim _{t \rightarrow \infty} \int_{0}^{t} \frac{s}{e^{t}} d s=0 .
\end{aligned}
$$

Furthermore, for any $x, y, z \in \mathrm{BC}\left(\mathbb{R}_{+}\right) \times \mathrm{BC}\left(\mathbb{R}_{+}\right) \times \mathrm{BC}\left(\mathbb{R}_{+}\right)$,

$$
\begin{aligned}
& \left|\int_{0}^{t} h(t, s, x(\eta(s)), y(\eta(s)), z(\eta(s))) d s\right| \\
& \quad \leqslant \int_{0}^{t}|h(t, s, x(\eta(s)), y(\eta(s)), z(\eta(s)))| d s \\
& \quad \leqslant \int_{0}^{t}\left(\frac{s}{2 e^{t}}+\frac{e^{s}}{e^{t}}\right) d s=\frac{t^{2}}{4 e^{t}}+1-\frac{1}{e^{t}} \\
& \quad=\frac{t^{2}+4 e^{t}-4}{4 e^{t}} .
\end{aligned}
$$

Thus,

$$
\begin{aligned}
& \sup \left\{\left|\int_{0}^{t} h(t, s, x(\eta(s)), y(\eta(s)), z(\eta(s))) d s\right|\right. \\
& \left.\quad t, s \in \mathbb{R}_{+}, x, y, z \in \mathrm{BC}\left(\mathbb{R}_{+}\right) \times \mathrm{BC}\left(\mathbb{R}_{+}\right) \times \mathrm{BC}\left(\mathbb{R}_{+}\right)\right\} \\
& =\sup \left\{\frac{t^{2}+4 e^{t}-4}{4 e^{t}}, t \in \mathbb{R}_{+}\right\}=1 .
\end{aligned}
$$

It is easy to see that, for any $r>0$, we have that

$$
\frac{1}{3} \varphi(3 r)+M_{1}+\Phi(\delta D) \leqslant r
$$

holds and condition (v) is valid.

Consequently, the system has at least one solution in $\mathrm{BC}\left(\mathbb{R}_{+}\right) \times \mathrm{BC}\left(\mathbb{R}_{+}\right) \times \mathrm{BC}\left(\mathbb{R}_{+}\right)$.

\section{Conflict of Interests}

The authors declare that there is no conflict of interests regarding the publication of this paper.

\section{References}

[1] K. Kuratowski, "Sur les espaces complets," Fundamenta Mathematicae, vol. 15, pp. 301-309, 1930.

[2] J. Banaś and K. Goebel, Measures of noncompactness in Banach Spaces, vol. 60 of Lecture Notes in Pure and Applied Mathematics, Marcel Dekker, New York, NY, USA, 1980.

[3] R. Agarwal, M. Meehan, and D. O'Regan, Fixed Point Theory and Applications, Cambridge University Press, 2004.

[4] A. Aghajani, J. Banaś, and Y. Jalilian, "Existence of solutions for a class of nonlinear Volterra singular integral equations," Computers \& Mathematics with Applications, vol. 62, no. 3, pp. 1215-1227, 2011.
[5] A. Aghajani and Y. Jalilian, "Existence and global attractivity of solutions of a nonlinear functional integral equation," Communications in Nonlinear Science and Numerical Simulation, vol. 15, no. 11, pp. 3306-3312, 2010.

[6] J. Banaś, "Measures of noncompactness in the study of solutions of nonlinear differential and integral equations," Central European Journal of Mathematics, vol. 10, no. 6, pp. 2003-2011, 2012.

[7] J. Banaś and B. Rzepka, "An application of a measure of noncompactness in the study of asymptotic stability", Applied Mathematics Letters, vol. 16, no. 1, pp. 1-6, 2003.

[8] M. Mursaleen and S. A. Mohiuddine, "Applications of measures of noncompactness to the infinite system of differential equations in $l_{p}$ space," Nonlinear Analysis: Theory, Methods \& Applications, vol. 75, no. 4, pp. 2111-2115, 2012.

[9] A. Aghajani, R. Allahyari, and M. Mursaleen, "A generalization of Darbo's theorem with application to the solvability of systems of integral equations," Journal of Computational and Applied Mathematics, vol. 260, pp. 68-77, 2014.

[10] J. Banaś, "On measures of noncompactness in Banach spaces," Commentationes Mathematicae Universitatis Carolinae, vol. 21, no. 1, pp. 131-143, 1980.

[11] S. S. Chang, Y. J. Cho, and N. J. Huang, "Coupled fixed point theorems with applications," Journal of the Korean Mathematical Society, vol. 33, no. 3, pp. 575-585, 1996.

[12] R. R. Akhmerov, M. I. Kamenski, A. S. Potapov, A. E. Rodkina, and B. N. Sadovski, Measures of Noncompactness and Condensing Operators, vol. 55, Birkhauser, Basel, Switzerland, 1992.

[13] A. Aghajani and N. Sabzali, "Existence of coupled fixed points via measure of noncompactness and applications," Journal of Nonlinear and Convex Analysis, vol. 15, no. 5, pp. 953-964, 2014.

[14] V. Berinde and M. Borcut, "Tripled fixed point theorems for contractive type mappings in partially ordered metric spaces," Nonlinear Analysis: Theory, Methods \& Applications, vol. 74, no. 15, pp. 4889-4897, 2011. 


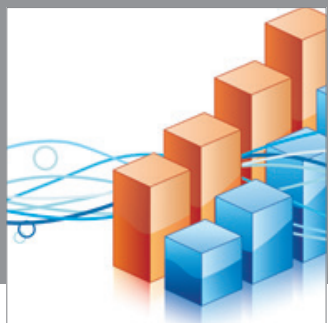

Advances in

Operations Research

mansans

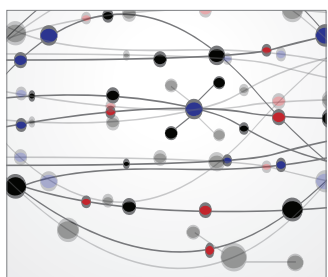

The Scientific World Journal
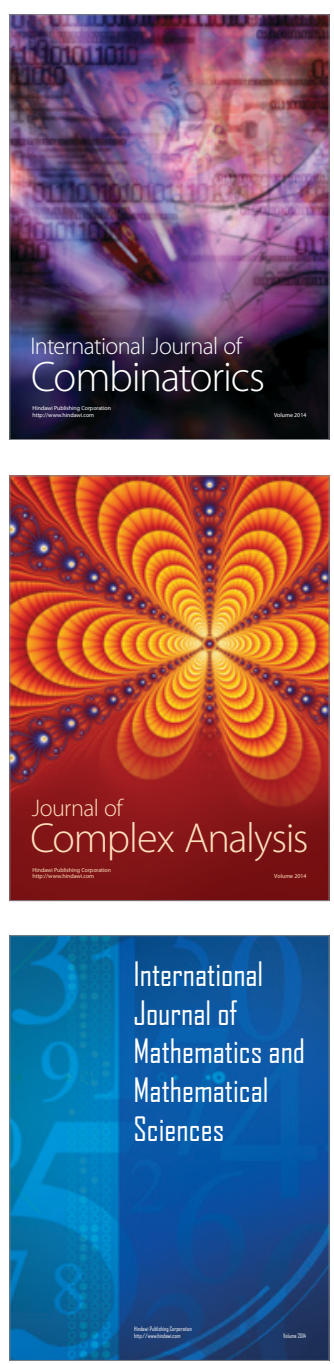
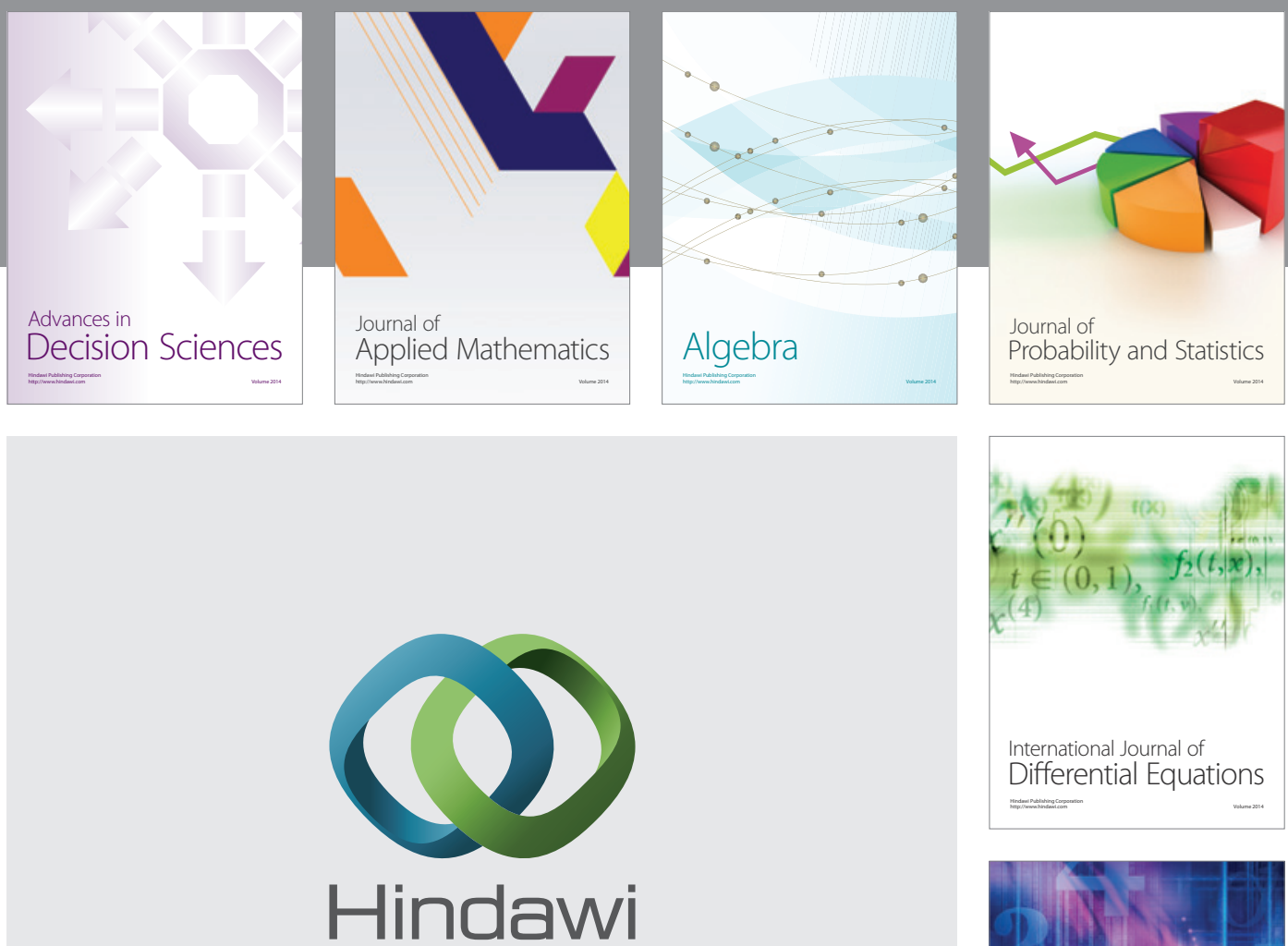

Submit your manuscripts at http://www.hindawi.com
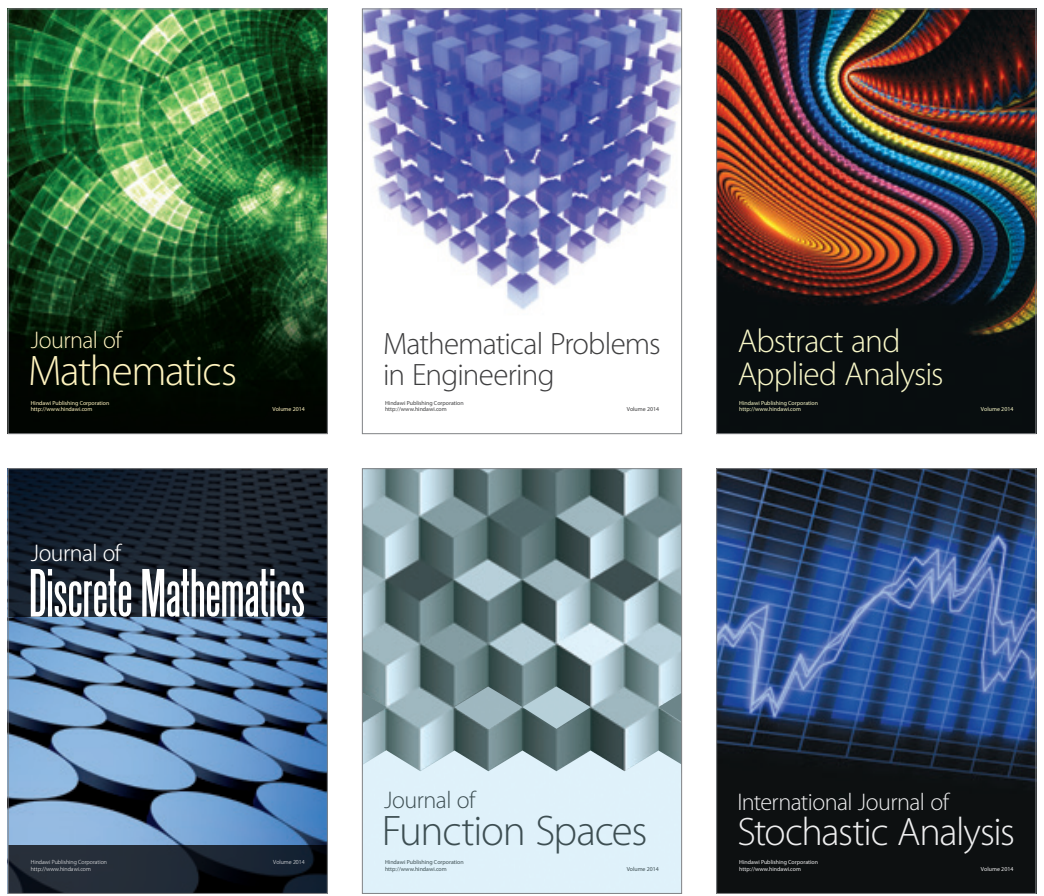

Journal of

Function Spaces

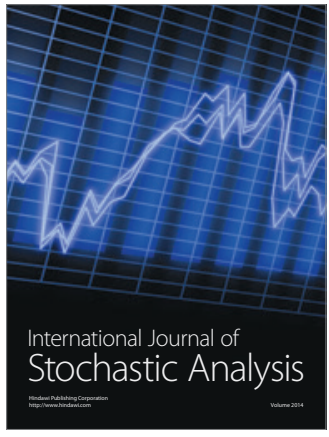

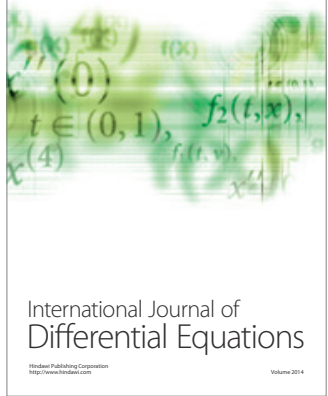
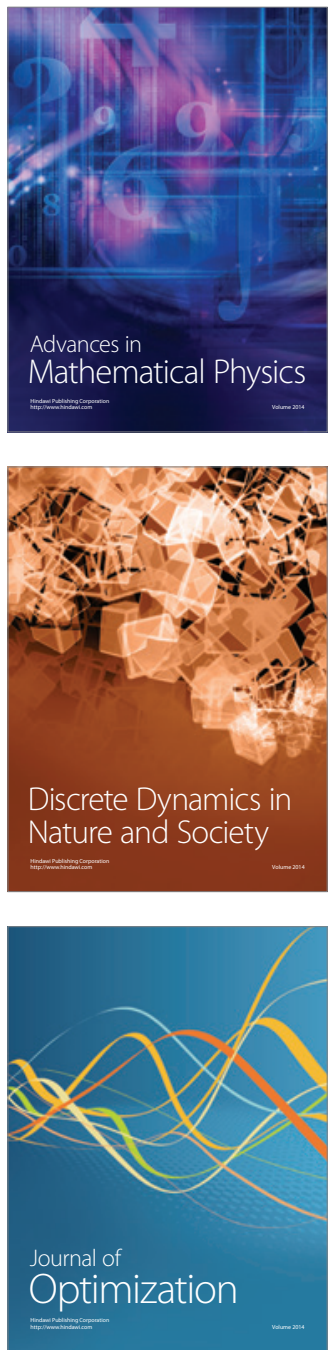